\title{
Highly Regioselective anti-Markovnikov Palladium-Borates Catalyzed Methoxycarbonylation Reactions: Unprecedented Results for Aryl Olefins
}

\author{
Tiago O. Vieira, ${ }^{\dagger}$ Mike J. Green ${ }^{\ddagger}$ and Howard Alper ${ }^{\dagger *}$ \\ ${ }^{\dagger}$ Contribution from the Centre for Catalysis Research and Innovation, Department of Chemistry, \\ University of Ottawa, 10 Marie Curie, Ottawa, Ontario, Canada K1N 6N5 \\ *asol Technology, 1 Klasie Havenga Road, Sasolburg 1947, South Africa \\ e-mail: howard.alper@uottawa.ca
}

\section{Supporting Information}

\section{General:}

The olefins were purchased from Aldrich or Alfa Aesar, tris-p-tolylphosphine from Lancaster and $\mathrm{Pd}(\mathrm{OAc})_{2}$ from Stream and were used without prior purification. Methanol was not distilled before use. Flash column chromatography was undertaken with silica gel (60 ^, 200-425 mesh) supplied by Aldrich. GC analyses were carried out using an Agilent 6850 series chromatograph. IR spectra were obtained with a Shimadzu FTIR-8400S spectrometer. Mass spectra were determined using a VG 7070E spectrometer. Solution ${ }^{1} \mathrm{H}$ NMR and ${ }^{13} \mathrm{C}$ NMR were recorded in $\mathrm{CDCl}_{3}$, containing TMS as internal standard, on a Bruker Avance $300 \mathrm{MHz}$. Chemical shifts $(\delta)$ are reported in ppm with the solvent signals as reference $\left({ }^{1} \mathrm{H}\right.$ NMR at $7.26 \mathrm{ppm}$ and ${ }^{13} \mathrm{C}$ NMR at 77.1 ppm), and coupling constants $(J)$ are given in Hertz (Hz).

Full spectroscopic and spectrometric characterization is provided only for new compounds or those which date are not available in the literature or described incompletely.

Carbon monoxide is a powerful asphyxiant and should be used with care. To use and work with carbon monoxide safely, reactions must be carried out in a proper working fumehood with carbon monoxide detectors installed nearby.

\section{General Procedure for the Methoxycarbonylation:}

A glass liner containing the olefin (2.00 mmol), 5-chlorosalicylic acid $(0.103 \mathrm{~g}, 0.600 \mathrm{mmol})$, boric acid (18.5 mg, $0.300 \mathrm{mmol})$, tris-p-tolylphosphine (0.0916 g, $0.300 \mathrm{mmol}), \mathrm{Pd}(\mathrm{OAc})_{2}(6.6 \mathrm{mg}, 0.030 \mathrm{mmol})$ and methanol $(3 \mathrm{~mL})$ was placed, in this order, in a $45 \mathrm{~mL}$ autoclave equipped with a magnetic stirring bar. The autoclave was flushed three times with carbon monoxide and pressurized to 400-600 psi, depending on the 
substrate. The autoclave was then placed in an oil bath pre-set to the desired temperature on a stirring hot plate (100 ${ }^{\circ} \mathrm{C}$ for the aliphatic olefins and $110{ }^{\circ} \mathrm{C}$ for the aromatic olefins). After completion of the reaction, the autoclave was removed from the oil bath and cooled to room temperature prior to the release of excess carbon monoxide. The reaction mixture was diluted with $\mathrm{Et}_{2} \mathrm{O}(100 \mathrm{~mL})$, was successively washed with $\mathrm{H}_{2} \mathrm{O}$ and brine and dried over $\mathrm{MgSO}_{4}$. After evaporation of the solvent, the residue was purified by silica gel chromatography with a mixture of hexane and ethyl acetate $(9: 1)$ as the eluant to afford the desired products.

\section{Compounds Prepared:}

$\Upsilon_{\gamma_{6}} \mathrm{CO}_{2} \mathrm{Me}$ Undecanoic acid methyl ester: ${ }^{1}$ colorless oil; $92 \% ;{ }^{1} \mathrm{H}$ NMR $\left(300 \mathrm{MHz}, \mathrm{CDCl}_{3}\right) \delta 0.88(\mathrm{t}, J=$ $6.9 \mathrm{~Hz}, 3 \mathrm{H}$ ), 1.26 (bs, 14H), 1.62 (quintet, $J=7.2 \mathrm{~Hz}, 2 \mathrm{H}), 2.30(\mathrm{t}, J=7.2 \mathrm{~Hz}, 2 \mathrm{H}), 3.67(\mathrm{~s}, 3 \mathrm{H})$.

$\checkmark \mathrm{T}_{10} \mathrm{CO}_{2} \mathrm{Me}$ Pentadecanoic acid methyl ester: ${ }^{2}$ colorless oil; $60 \% ;{ }^{1} \mathrm{H} \mathrm{NMR}\left(300 \mathrm{MHz}, \mathrm{CDCl}_{3}\right) \delta 0.88(\mathrm{t}, J=$ $6.9 \mathrm{~Hz}, 3 \mathrm{H}$ ), 1.26 (bs, 22H), 1.62 (quintet, $J=7.2 \mathrm{~Hz}, 2 \mathrm{H}), 2.30(\mathrm{t}, J=7.2 \mathrm{~Hz}, 2 \mathrm{H}), 3.67(\mathrm{~s}, 3 \mathrm{H})$.

$\mathrm{CO}_{2} \mathrm{Me}$ 4-Cyclopentyl-butyric acid methyl ester: ${ }^{3}$ colorless oil; 70\%; ${ }^{1} \mathrm{H} \mathrm{NMR}\left(300 \mathrm{MHz}, \mathrm{CDCl}_{3}\right)$ $\delta$ 1.03-1.78 (m, 13H), $2.30(\mathrm{t}, J=7.5 \mathrm{~Hz}, 2 \mathrm{H}), 3.66(\mathrm{~s}, 3 \mathrm{H}) ;{ }^{13} \mathrm{C} \mathrm{NMR}\left(75.4 \mathrm{MHz}, \mathrm{CDCl}_{3}\right) \delta$ 24.2, 25.2, 32.6, 34.4, $35.7,39.9,51.5,174.4$.

4-Methyl-heptanoic acid methyl ester: ${ }^{4}$ colorless oil; 75\%; ${ }^{1} \mathrm{H} \mathrm{NMR}\left(300 \mathrm{MHz}, \mathrm{CDCl}_{3}\right) \delta$ $\mathrm{CO}_{2} \mathrm{Me} \quad 0.84(\mathrm{~d}, J=7.4 \mathrm{~Hz}, 3 \mathrm{H}), 0.86(\mathrm{~d}, J=6.6 \mathrm{~Hz}, 3 \mathrm{H}), 0.90-1.68(\mathrm{~m}, 7 \mathrm{H}), 2.28-2.41(\mathrm{~m}, 2 \mathrm{H}), 3.66(\mathrm{~s}$, $3 \mathrm{H}) ;{ }^{13} \mathrm{C} \mathrm{NMR}\left(75.4 \mathrm{MHz}, \mathrm{CDCl}_{3}\right) \delta 14.3,19.2,20.0,21.3,31.9,32.1,38.9,51.5,174.6$.

$\mathrm{CO}_{2} \mathrm{Me}$ 4-Phenyl-butyric acid methyl ester: ${ }^{5}$ colorless oil; 80\%; ${ }^{1} \mathrm{H}$ NMR $\left(300 \mathrm{MHz}, \mathrm{CDCl}_{3}\right) \delta 1.95$ (quintet, $J=7.5 \mathrm{~Hz}, 2 \mathrm{H}), 2.32(\mathrm{t}, J=7.5 \mathrm{~Hz}, 2 \mathrm{H}), 2.64(\mathrm{t}, J=7.5 \mathrm{~Hz}, 2 \mathrm{H}), 3.65(\mathrm{~s}, 3 \mathrm{H}), 7.16-7.30$ $(\mathrm{m}, 5 \mathrm{H}) ;{ }^{13} \mathrm{C} \mathrm{NMR}\left(75.4 \mathrm{MHz}, \mathrm{CDCl}_{3}\right) \delta 26.5,33.3,35.1,51.5,126.0,126.3,128.4,128.5,173.9$.

$\mathrm{CO}_{2} \mathrm{Me}$ 4-(4-Methoxy-phenyl)-butyric acid methyl ester: ${ }^{6}$ yellowish oil; 75\%; ${ }^{1} \mathrm{H}$ NMR $(300$
$\left.\mathrm{MHz}, \mathrm{CDCl}_{3}\right) \delta 1.91$ (quintet, $\left.J=7.5 \mathrm{~Hz}, 2 \mathrm{H}\right), 2.31(\mathrm{t}, J=7.5 \mathrm{~Hz}, 2 \mathrm{H}), 2.58(\mathrm{t}, J=7.5 \mathrm{~Hz}$,

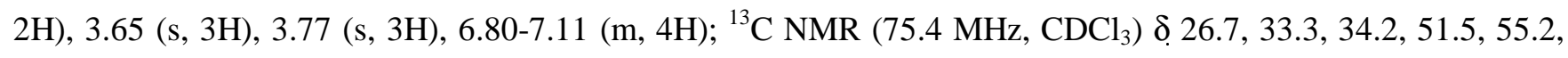
$113.7,129.4,133.4,157.9,174.0$.

\footnotetext{
${ }^{1}$ Kling, M. R.; Easton, C. J.; Poulos, A. J. Chem. Soc. Perkin Trans. 1 1993, 1183.

${ }^{2}$ Yu, J. G.; Ho, D. K.; Cassady, J. M.; Xu, L.; Chang, C. -I. J. Org. Chem.1993, 57, 6198.

${ }^{3}$ Giese, B.; Kretzschmar, G Chem. Ber. 1984, 117, 3160.

${ }^{4}$ Casey, C. P.; Cyr, C. R. J. Am. Chem. Soc. 1973, 95, 2240.

${ }^{5}$ Inaba, S. -I.; Matsumoto, H.; Rieke, R. D. J. Org. Chem. 1984, 49, 2093.

${ }^{6}$ Chenal, T.; Cipres, I.; Jenck, J.; Kalck, P. J. Mol. Cat. 1993, 78, 351.
} 


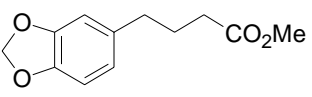

4-Benzo[1,3]dioxol-5-yl-butyric acid methyl ester: ${ }^{7}$ yellowish oil; 74\%; ${ }^{1} \mathrm{H}$ NMR (300 $\mathrm{MHz}, \mathrm{CDCl}_{3}$ ) $\delta 1.90$ (quintet, $J=7.5 \mathrm{~Hz}, 2 \mathrm{H}$ ), $2.31(\mathrm{t}, J=7.5 \mathrm{~Hz}, 2 \mathrm{H}), 2.56(\mathrm{t}, J=7.5 \mathrm{~Hz}$, 2H), 3.66 (s, 3H), 5.91 (s, 2H), 6.60-6.73 (m, 3H); $\left.{ }^{13} \mathrm{C} \mathrm{NMR} \mathrm{(75.4} \mathrm{MHz,} \mathrm{CDCl}_{3}\right) \delta$ 26.7, 33.2, 34.8, 51.5, 100.8, $108.2,108.9,121.3,135.2,145.7,147.6,173.9$.

$\mathrm{CO}_{2} \mathrm{Me}$ 3-Phenyl-propionic acid methyl ester: ${ }^{8}$ colorless oil; $66 \% ;{ }^{1} \mathrm{H}$ NMR $\left(300 \mathrm{MHz}, \mathrm{CDCl}_{3}\right) \delta 2.63$ (t, $J=7.5 \mathrm{~Hz}, 2 \mathrm{H}), 2.95(\mathrm{t}, J=7.5 \mathrm{~Hz}, 2 \mathrm{H}), 3.66(\mathrm{~s}, 3 \mathrm{H}), 7.17-7.31(\mathrm{~m}, 5 \mathrm{H}) ;{ }^{13} \mathrm{C} \mathrm{NMR}(75.4 \mathrm{MHz}$, $\left.\mathrm{CDCl}_{3}\right) \delta 30.9,35.7,51.6,126.3,128.3,128.5,140.5,173.3$.

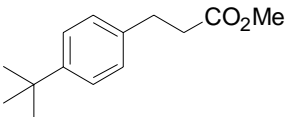

3-(4-tert-Butyl-phenyl)-propionic acid methyl ester: colorless oil; 91\%; IR (neat) 1736 $\mathrm{cm}^{-1} ;{ }^{1} \mathrm{H}$ NMR $\left(300 \mathrm{MHz}, \mathrm{CDCl}_{3}\right) \delta 1.30(\mathrm{~s}, 9 \mathrm{H}), 2.62(\mathrm{t}, J=7.5 \mathrm{~Hz}, 2 \mathrm{H}), 2.92(\mathrm{t}, J=7.5 \mathrm{~Hz}$, 2H), 3.67 (s, 3H), 7.12-7.32 (m, 4H); ${ }^{13} \mathrm{C}$ NMR (75.4 MHz, $\mathrm{CDCl}_{3}$ ) $\delta$ 30.4, 31.4, 34.4, 35.7, 51.7, 125.4, 127.9, 137.4, 149.1, 173.5. HRMS Calcd. for $\mathrm{C}_{14} \mathrm{H}_{20} \mathrm{O}_{2} 220.1463$, found 220.1456 .

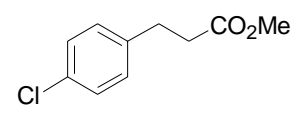

3-(4-Chloro-phenyl)-propionic acid methyl ester: ${ }^{9}$ colorless oil; 72\%; ${ }^{1} \mathrm{H}$ NMR $(300 \mathrm{MHz}$, $\left.\mathrm{CDCl}_{3}\right) \delta 2.60(\mathrm{t}, J=7.5 \mathrm{~Hz}, 2 \mathrm{H}), 2.91(\mathrm{t}, J=7.5 \mathrm{~Hz}, 2 \mathrm{H}), 3.66(\mathrm{~s}, 3 \mathrm{H}), 7.12(\mathrm{~d}, J=8.5 \mathrm{~Hz}$, 2H), $7.24(\mathrm{~d}, J=8.5 \mathrm{~Hz}, 2 \mathrm{H}) ;{ }^{13} \mathrm{C} \mathrm{NMR}\left(75.4 \mathrm{MHz}, \mathrm{CDCl}_{3}\right) \delta 30.2,35.5,51.7,128.6,129.7,132.1,139.0,173.1$.

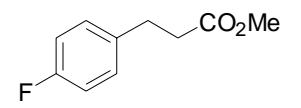

3-(4-Fluoro-phenyl)-propionic acid methyl ester: colorless oil; 72\%; IR (neat) $1732 \mathrm{~cm}^{-1}$; ${ }^{1} \mathrm{H}$ NMR (300 MHz, $\left.\mathrm{CDCl}_{3}\right) \delta 2.60$ (t, $\left.J=7.6 \mathrm{~Hz}, 2 \mathrm{H}\right), 2.92$ (t, $\left.J=7.6 \mathrm{~Hz}, 2 \mathrm{H}\right), 3.66(\mathrm{~s}, 3 \mathrm{H})$, 6.93-7.17 (m, 4H); ${ }^{13} \mathrm{C}$ NMR (75.4 MHz, $\left.\mathrm{CDCl}_{3}\right) \delta 30.1,35.8,51.7,115.1,115.4,129.8(\mathrm{~d}, J=7.9 \mathrm{~Hz}), 136.1(\mathrm{~d}$,

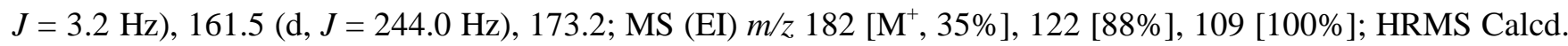
for $\mathrm{C}_{10} \mathrm{H}_{11} \mathrm{O}_{2} \mathrm{~F}$ 182.0743, found 187.0741.

$\mathrm{CO}_{2} \mathrm{Me}$

3-o-Tolyl-propionic acid methyl ester: colorless oil; 60\%; IR (neat) $1736 \mathrm{~cm}^{-1}$; ${ }^{1} \mathrm{H}$ NMR (300 $\left.\mathrm{MHz}, \mathrm{CDCl}_{3}\right) \delta 2.32(\mathrm{~s}, 3 \mathrm{H}), 2.59$ (t, $\left.J=7.5 \mathrm{~Hz}, 2 \mathrm{H}\right), 2.94(\mathrm{t}, J=7.5 \mathrm{~Hz}, 2 \mathrm{H}), 3.68(\mathrm{~s}, 3 \mathrm{H}), 7.13$ $(\mathrm{s}, 4 \mathrm{H}) ;{ }^{13} \mathrm{C} \mathrm{NMR}\left(75.4 \mathrm{MHz}, \mathrm{CDCl}_{3}\right) \delta$ 19.3, 28.4, 34.5, 51.7, 126.2, 126.5, 128.5, 130.4, 136.0, 138.6, 173.5; HRMS Calcd. for $\mathrm{C}_{11} \mathrm{H}_{14} \mathrm{O}_{2}$ 178.0994, found 178.1002.

$\mathrm{CO}_{2} \mathrm{Me}$ 3-(2-Bromo-phenyl)-propionic acid methyl ester: ${ }^{10}$ colorless oil; $65 \%$; IR (neat) $1732 \mathrm{~cm}^{-1} ;{ }^{1} \mathrm{H}$ $\mathrm{Br}$ NMR (300 MHz, $\left.\mathrm{CDCl}_{3}\right) \delta 2.65(\mathrm{t}, J=7.5 \mathrm{~Hz}, 2 \mathrm{H}), 3.06(\mathrm{t}, J=7.5 \mathrm{~Hz}, 2 \mathrm{H}), 3.67(\mathrm{~s}, 3 \mathrm{H}), 7.04-$

\footnotetext{
${ }^{7}$ Esteban, G.; López-Sánchez, M. A.; Martínez, M. E.; Plunet, J. Tetrahedron 1998, 54, 197.

${ }^{8}$ Semmelhack, M. F.; Stauffer, R. D.; Yamashita, A. J. Org. Chem. 1977, 42, 3180.

${ }^{9}$ Louie, J.; Bielawski, C. W.; Grubbs, R. H. J. Am. Chem. Soc. 2001, 123, 11312.

${ }^{10}$ Beak, P.; Selling, G. W. J. Org. Chem. 1989, 54, 5574.
} 
$7.26(\mathrm{~m}, 3 \mathrm{H}), 7.52(\mathrm{~d}, J=7.8 \mathrm{~Hz}, 1 \mathrm{H}) ;{ }^{13} \mathrm{C} \mathrm{NMR}\left(75.4 \mathrm{MHz}, \mathrm{CDCl}_{3}\right) \delta .31 .4,33.9,51.7,124.3,127.6,128.1$, $130.4,132.9,139.7,173.0$.

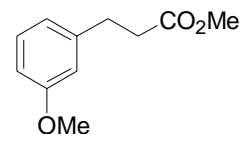

3-(3-Methoxy-phenyl)-propionic acid methyl ester: ${ }^{11}$ colorless oil; 84\%; ${ }^{1} \mathrm{H}$ NMR $(300 \mathrm{MHz}$, $\left.\mathrm{CDCl}_{3}\right) \delta 2.63$ (t, $\left.J=7.5 \mathrm{~Hz}, 2 \mathrm{H}\right), 2.93$ (t, $\left.J=7.5 \mathrm{~Hz}, 2 \mathrm{H}\right), 3.67$ (s, 3H), 3.79 (s, 3H), 6.74-6.81 (m, 3H), 7.17-7.25 (m, 1H); ${ }^{13} \mathrm{C} \mathrm{NMR}\left(75.4 \mathrm{MHz}, \mathrm{CDCl}_{3}\right) \delta 31.0,35.6,51.6,55.1,111.5,114.0,120.6,129.5,142.1$, 159.7, 173.3.

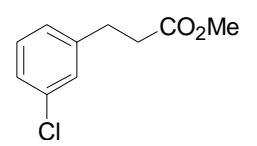

3-(3-Chloro-phenyl)-propionic acid methyl ester: colorless oil; 66\%; IR (neat) $1732 \mathrm{~cm}^{-1}$; ${ }^{1} \mathrm{H}$ NMR (300 MHz, $\left.\mathrm{CDCl}_{3}\right) \delta 2.62(\mathrm{t}, J=7.5 \mathrm{~Hz}, 2 \mathrm{H}), 2.93(\mathrm{t}, J=7.5 \mathrm{~Hz}, 2 \mathrm{H}), 3.67(\mathrm{~s}, 3 \mathrm{H}), 7.06-$ $7.22(\mathrm{~m}, 4 \mathrm{H}) ;{ }^{13} \mathrm{C}$ NMR $\left(75.4 \mathrm{MHz}, \mathrm{CDCl}_{3}\right) \delta$ 30.6, 35.4, 51.7, 125.5 (2 overlaped C), 128.5, 129.8, 134.2, 142.5, 173.0; Calcd. for $\mathrm{C}_{10} \mathrm{H}_{11} \mathrm{O}_{2} \mathrm{Cl} 198.0447$, found 198.0439.

3-Phenyl-butyric acid methyl ester: ${ }^{12}$ colorless oil; 74\%; ${ }^{1} \mathrm{H}$ NMR $\left(300 \mathrm{MHz}, \mathrm{CDCl}_{3}\right) \delta 1.29(\mathrm{~d}$, $J=7.0 \mathrm{~Hz}, 3 \mathrm{H}), 2.54(\mathrm{dd}, J=15.1$ and $8.2 \mathrm{~Hz}, 1 \mathrm{H}), 2.63(\mathrm{dd}, J=15.1$ and $6.9 \mathrm{~Hz}, 1 \mathrm{H}), 3.22-3.36$

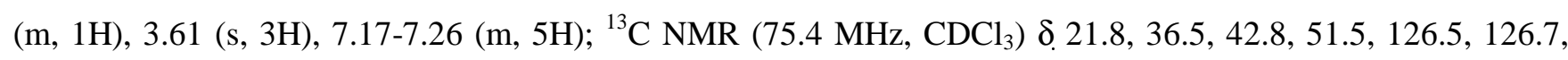
$128.5,145.7,172.9$.

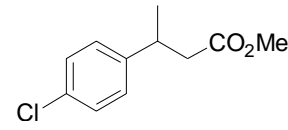

3-(4-Chloro-phenyl)-butyric acid methyl ester: ${ }^{13}$ colorless oil; 71\%; ${ }^{1} \mathrm{H}$ NMR $(300 \mathrm{MHz}$, $\left.\mathrm{CDCl}_{3}\right) \delta 1.27(\mathrm{~d}, J=6.5 \mathrm{~Hz}, 3 \mathrm{H}), 2.52(\mathrm{dd}, J=15.3$ and $7.9 \mathrm{~Hz}, 1 \mathrm{H}), 2.59$ (dd, $J=15.3$ and $7.3 \mathrm{~Hz}, 1 \mathrm{H}), 3.20-3.32(\mathrm{~m}, 1 \mathrm{H}), 3.61(\mathrm{~s}, 3 \mathrm{H}), 7.11-7.27(\mathrm{~m}, 4 \mathrm{H}) ;{ }^{13} \mathrm{C} \mathrm{NMR}\left(75.4 \mathrm{MHz}, \mathrm{CDCl}_{3}\right) \delta 21.8,35.9,42.6$, $51.6,128.2,128.6,132.1,144.1,176.6$.

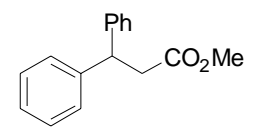

3,3-Diphenyl-propionic acid methyl ester: ${ }^{14}$ colorless oil; 73\%; ${ }^{1} \mathrm{H}$ NMR $\left(300 \mathrm{MHz}, \mathrm{CDCl}_{3}\right) \delta$ $3.05(\mathrm{~d}, J=8.0 \mathrm{~Hz}, 2 \mathrm{H}), 3.56(\mathrm{~s}, 3 \mathrm{H}), 4.56(\mathrm{t}, J=8.0 \mathrm{~Hz}, 1 \mathrm{H}), 7.15-7.26(\mathrm{~m}, 10 \mathrm{H}) ;{ }^{13} \mathrm{C} \mathrm{NMR}(75.4$ $\left.\mathrm{MHz}, \mathrm{CDCl}_{3}\right) \delta$ 40.6, 47.0, 51.7, 126.6, 127.7, 128.6, 143.5, 172.3.

\footnotetext{
${ }^{11}$ Hwang, K. -J.; O’Neil, J. P.; Katzenellenbogen, J. A. J. Org. Chem. 1992, 57, 1262.

${ }^{12}$ Humphrey, C. E.; Turner, N. J.; Easson, M. A. M.; Flitsch, S. L.; Ulijn, R. V. J. Am. Chem. Soc. 2003, 125, 13952.

${ }^{13}$ Citterio, A.; Minisci, F.; Vismara, E. J. Org. Cehm. 1982, 47, 81.

${ }^{14}$ Oi, S.; Moro, M.; Ito, H.; Honma, Y.; Miyano, S.; Inoue, Y. Tetrahedron 2002, 58, 91.
} 

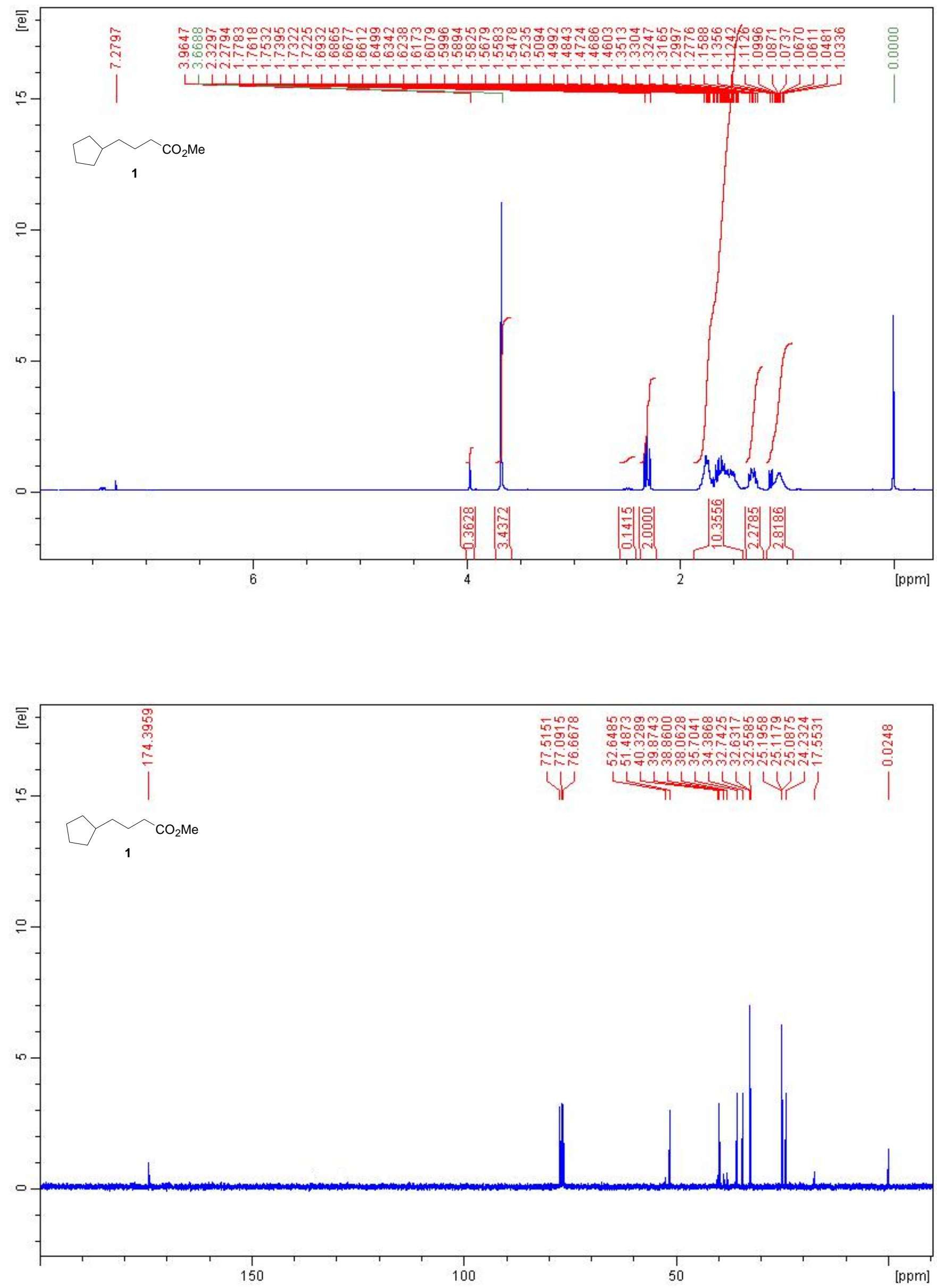

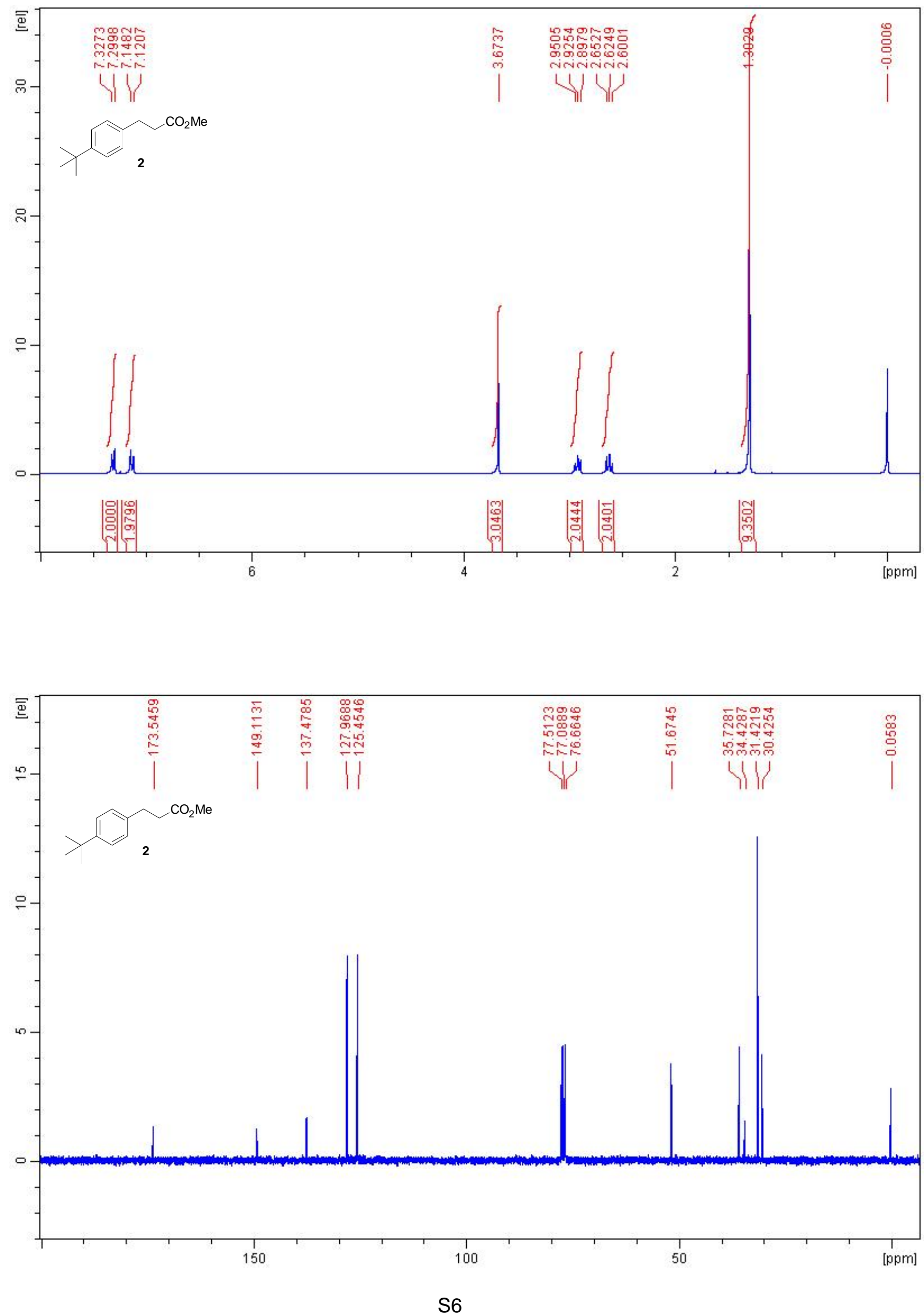

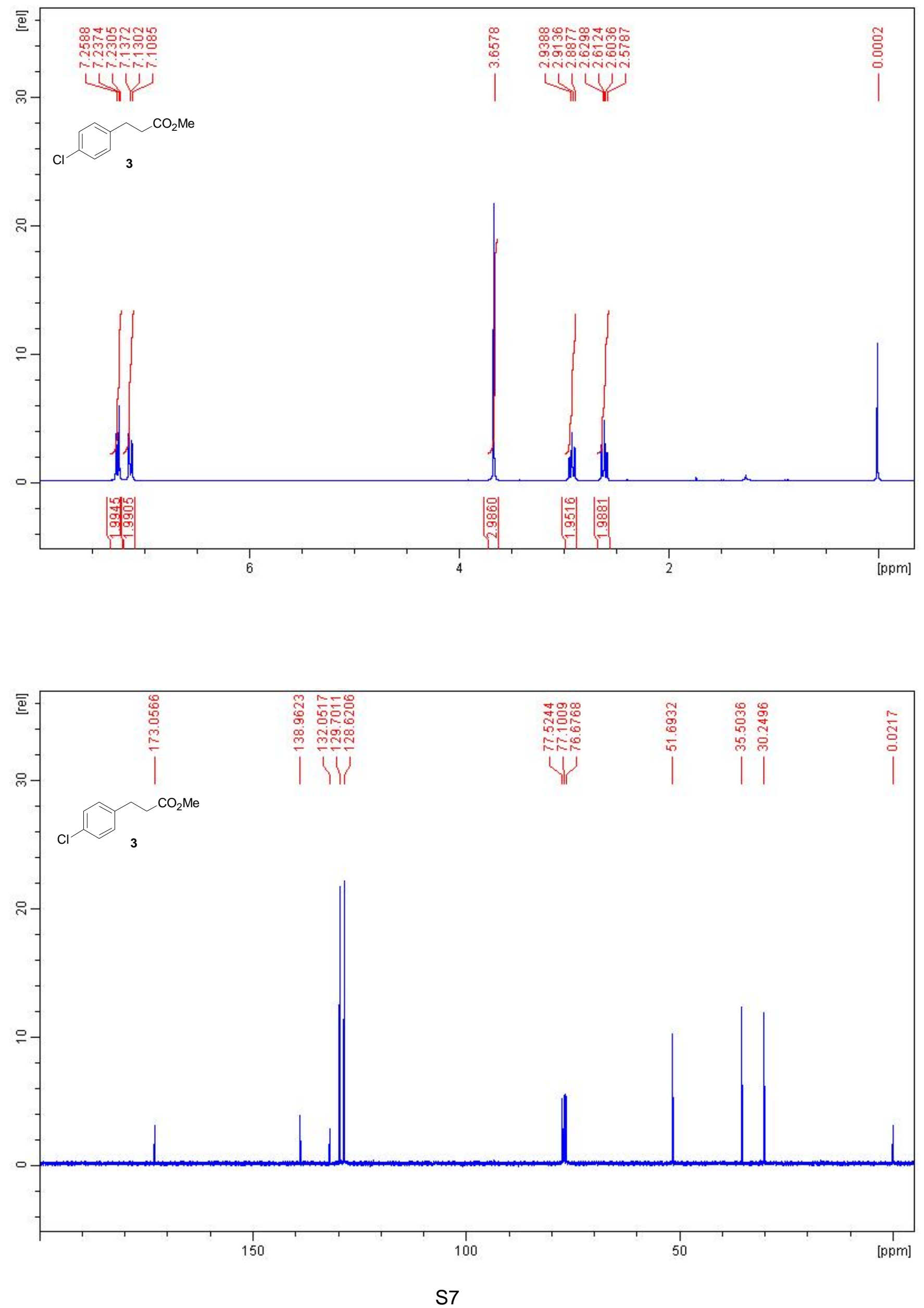

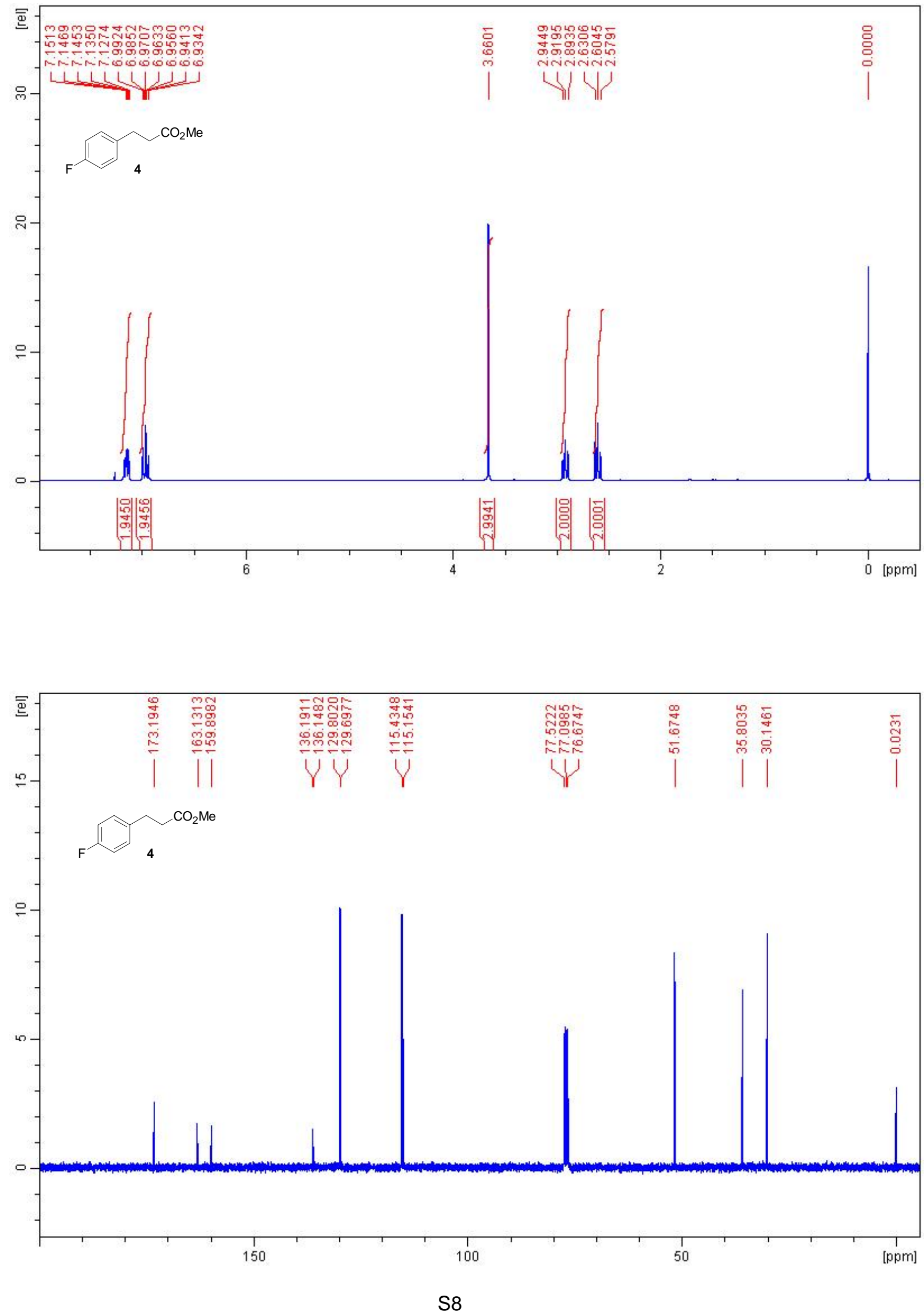

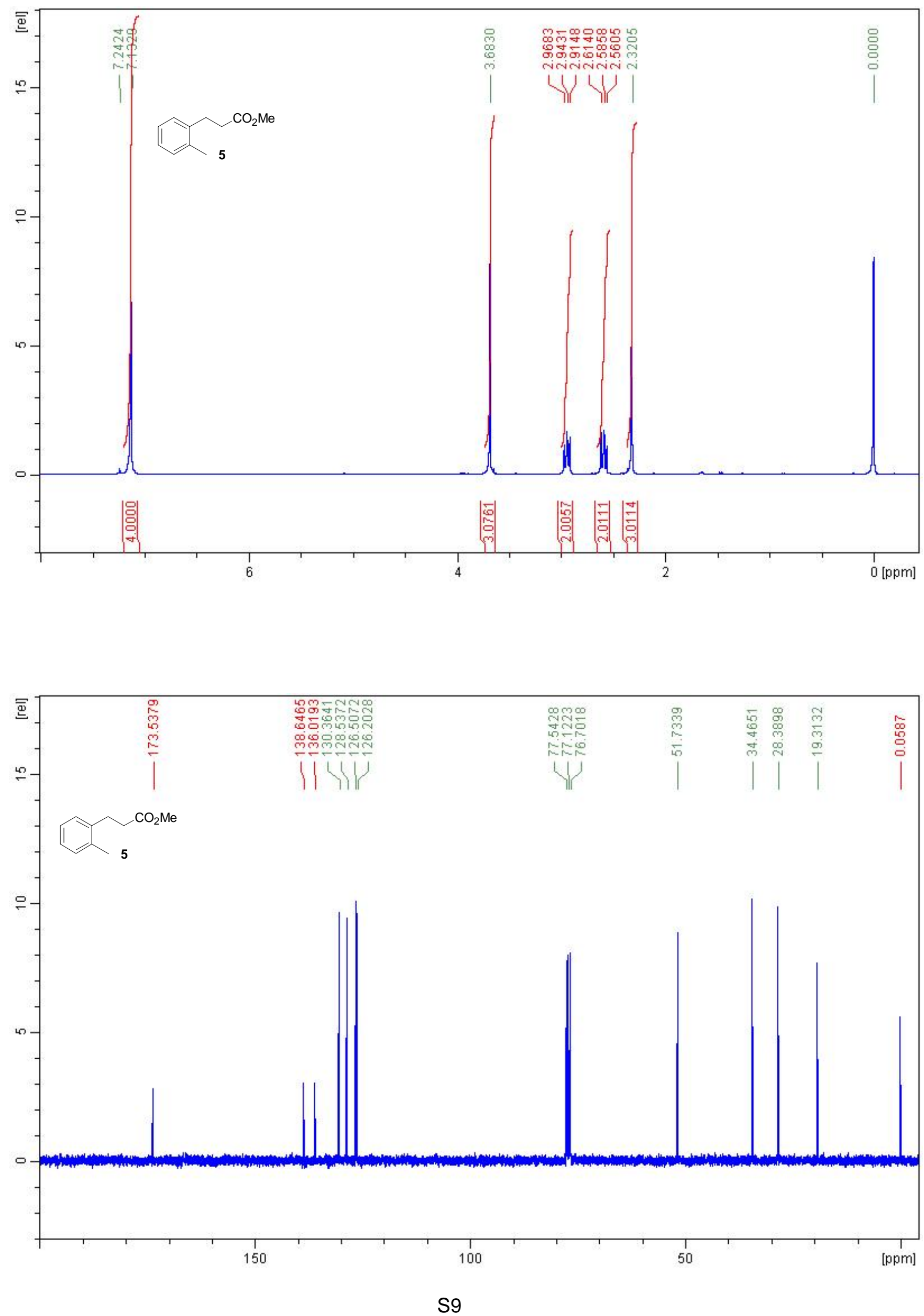

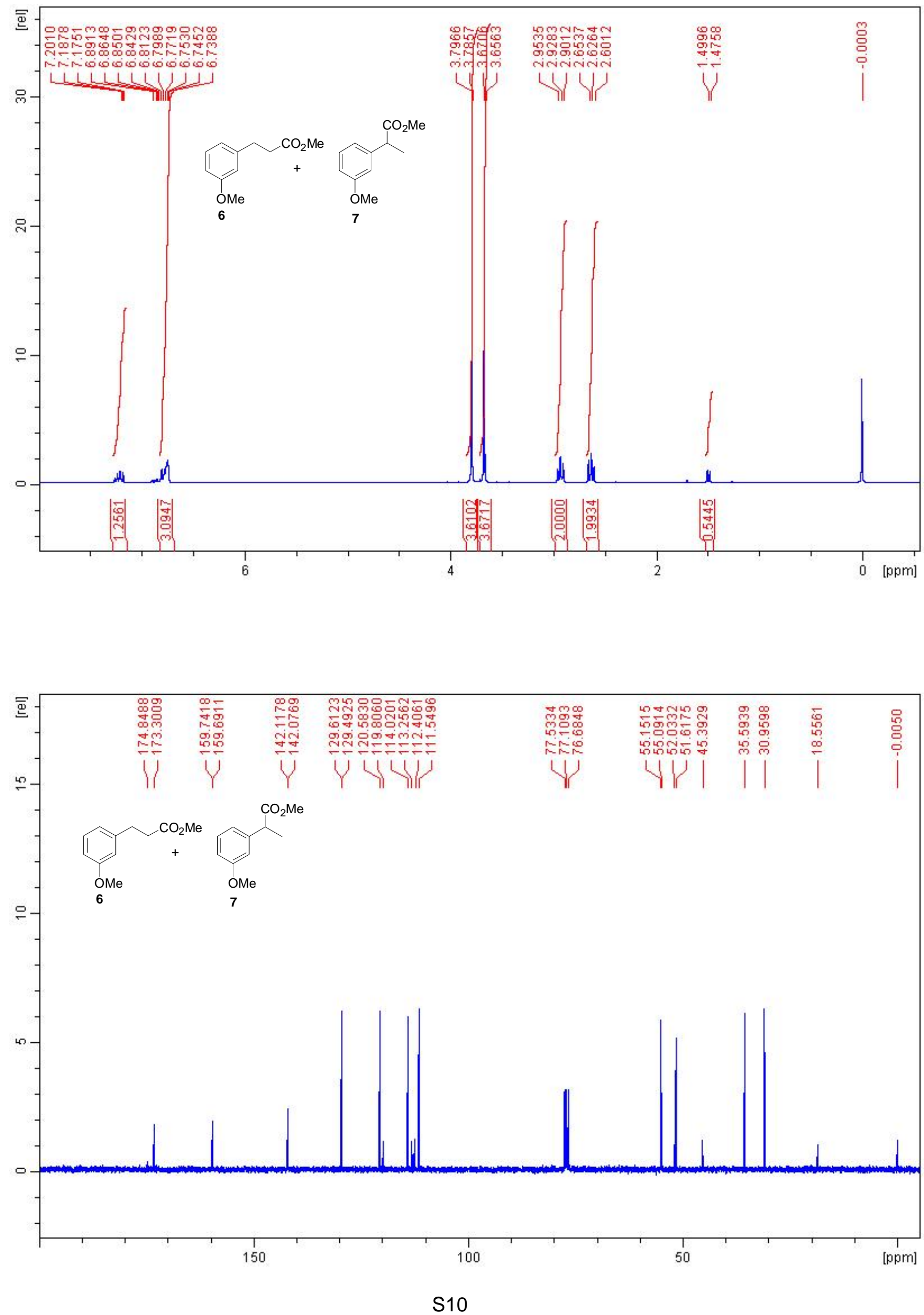

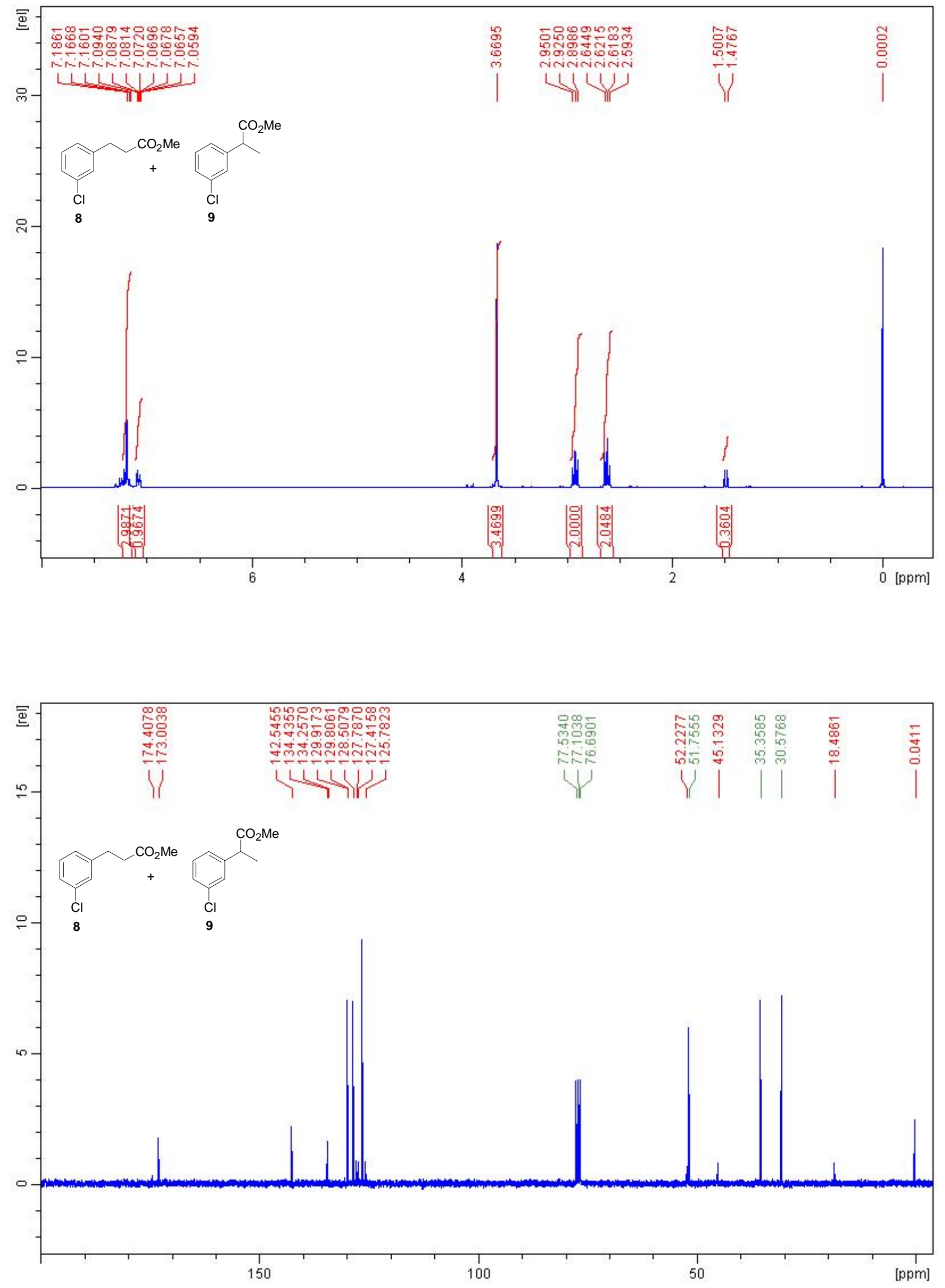

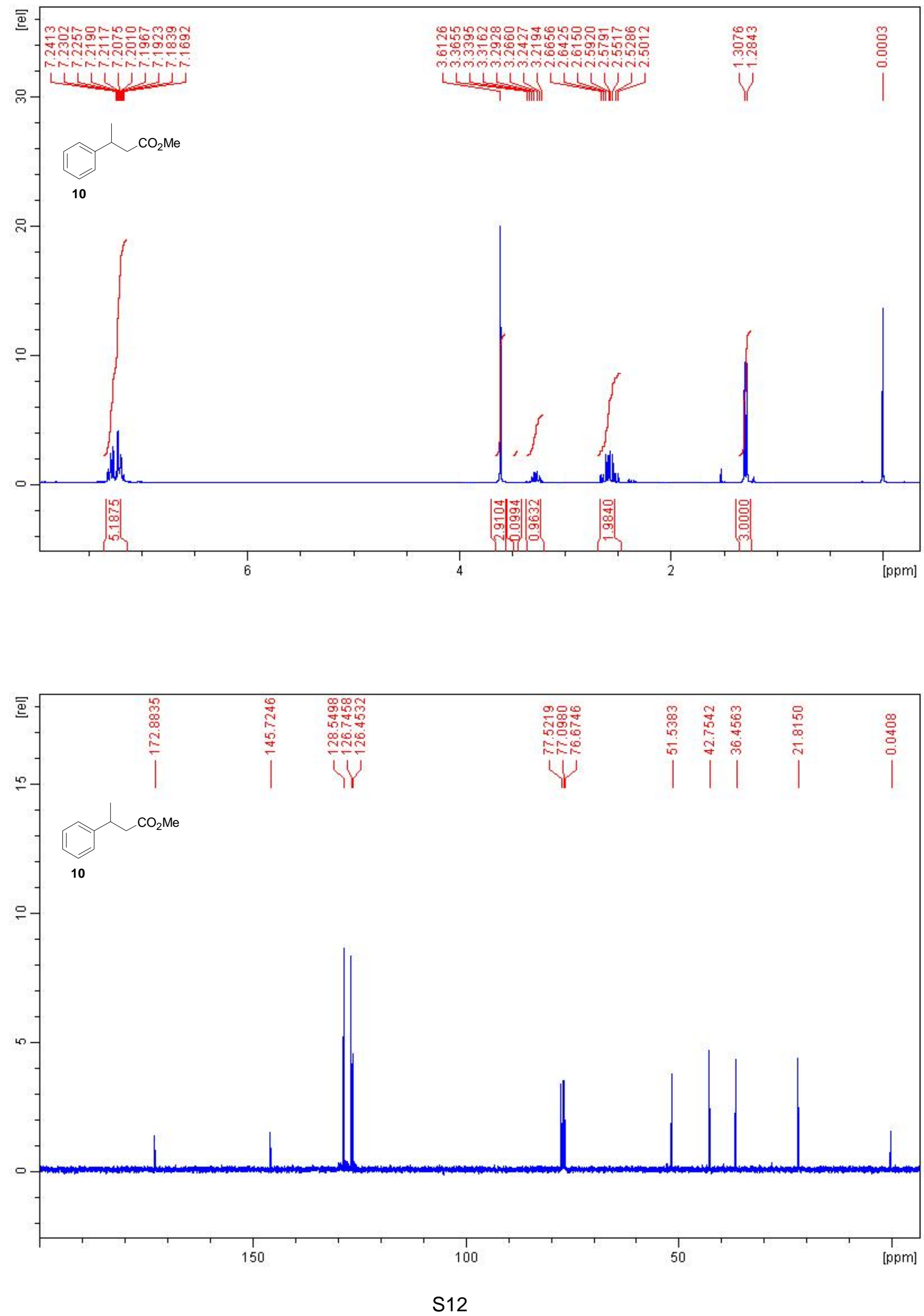

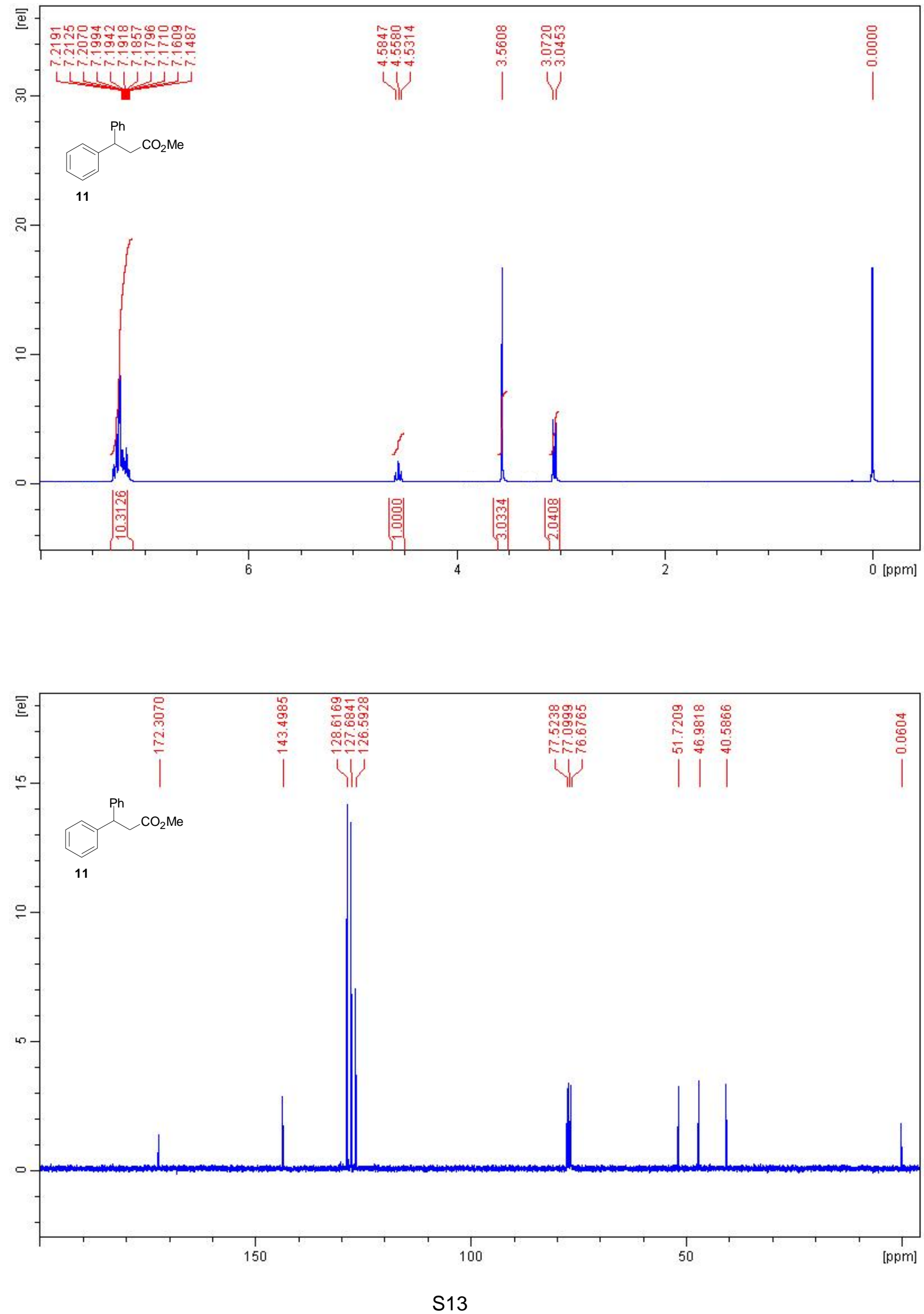